\title{
El interés por la botánica de Luis M. Ramírez de las Casas-Deza(1802-1874)
}

\author{
Juan Antonio Devesa ${ }^{1}$ y Ángel Montero² \\ 'Departamento de Botánica, Ecología y Fisiología Vegetal, Facultad de Ciencias, Universidad de Córdoba. Edificio José \\ Celestino Mutis, Campus de Rabanales, Universidad de Córdoba, 14071 (Córdoba). \\ 2Jardín Botánico de Córdoba, Avenida de Linneo s/n, 14004 (Córdoba).
}

\section{Correspondencia}

J. A. Devesa

e-mail: bv1dealj@uco.es

Recibido: 21 julio 2018

Aceptado: 21 septiembre 2018

Publicado on-line: septiembre 2018
Interest in the Botany of Luis M. Ramírez de las Casas-Deza (1802-1874)

Palabras clave. Ramírez de las Casas-Deza, historia, botánica, Córdoba.

Key words. Ramírez de las Casas-Deza, History, Botany, Córdoba.

\section{El personaje}

El cordobés Luis María Ramírez de las CasasDeza (1802-1874), aunque médico de profesión, es conocido sobre todo como humanista e historiador, si bien entre sus múltiples facetas relacionadas en su mayor parte con la cultura destaca una apenas conocida, la de su interés por la Botánica, que se comenta más adelante.

Ramírez de las Casas-Deza nació en Córdoba el 2 de junio de 1802, ciudad en la que inició su formación antes de desplazarse a Sevilla, en septiembre de 1822, para obtener el grado de Bachiller en Filosofía por la Universidad Literaria. Aquí comenzó también los estudios de Medicina, que culminó dos años más tarde. En septiembre de 1826 se desplazó a Madrid para cursar la materia Clínica en el Real Estudio de Medicina Práctica, bajo la supervisión de D. Antonio Hernández Morejón, Médico de Cámara de Su Majestad (Ramírez de las Casas-Deza, 1977), obteniendo el grado de Licenciado en Medicina ante la Junta Superior Gubernativa el 5 de diciembre de 1826. En la capital del reino compaginó sus estudios universitarios con el aprendizaje de Zoología en el Museo de Historia Natural durante el bienio 18261827, bajo la dirección de D. Tomás Villanova, Médico de Cámara de S.M., y también un curso de Botánica con aplicación a la Medicina, bajo la dirección de D. Vicente Soriano ("Don Vicente Soriano, que más adelante fue nombrado catedrático de Botánica General, nos explicó al final del año [1826] un curso de Botánica con aplicación a la Medicina"; Ramírez de las CasasDeza, 1977), botánico valenciano que desde
1817 se había hecho cargo de la enseñanza de Botánica medicinal en el Jardín Botánico de Madrid (López González, 2005). Su afición a la geografía empezó con dieciséis años, después de leer " $\mathrm{La}$ Clave Geográfica del P. Flórez", y con los años fue ampliando sus conocimientos, como él mismo señala en sus memorias (Ramírez de las CasasDeza, 1977).

Ejerció la actividad galénica en las poblaciones cordobesas de Bujalance, Villafranca, El Carpio y Pozoblanco, y en la de Fuencaliente, Ciudad Real (Borja Pavón, 1874; Ramírez de las Casas-Deza, 1977; López Ontiveros, 1984), con estancias temporales en otros lugares, incluyendo Córdoba, ciudad en la que no acababa de encontrar medios estables para su subsistencia, casi siempre muy precaria. Estas penurias económicas quedan crudamente subrayadas en sus escritos, como señala López Ontiveros (1984), citando a Ramírez de las Casas-Deza: "Al médico se acude aunque no haya con qué pagarle, y o no se les da nada, o se aplaza el satisfacerle para tal o cuál tiempo, lo que pocas veces o nunca llega, ya sea que el enfermo recobre la salud, ya que fallezca, que entonces es mas difícil la cobranza... Donde están dotados los médicos se les paga muy mal, donde están igualados tienen que ir a percibir las cuotas de puerta en puerta como quien pide limosna, y sin embargo de practicar esta diligencia por si mismo para obligar mas al pago llega al fin del año y la mayor parte de la deuda queda sin cobrar".

Todo cambió cuando en el año 1842 comenzó a regentar la cátedra de Geografía e Historia del Instituto de Segunda Enseñanza de Córdoba, actividad que se prolongaría durante 20 años. Aquí 
simultaneó la actividad docente con su verdadera vocación, la de escritor sobre temas muy diversos (literatura, historia, arqueología, etc.). Casas-Deza fue, probablemente, el geógrafo e historiador cordobés más destacado del siglo XIX, y a él se deben algunas obras de interés sobre la historia y características geográficas y paisajísticas de la ciudad de Córdoba y de su provincia, entre ellas su Indicador cordobés (Ramírez de las CasasDeza, 1837), Corografía Histórico-Estadística de la provincia y obispado de Córdoba (Ramírez de las Casas-Deza, 1840), Breve tratado de Geografía de la Provincia de Córdoba para uso de los colegios y escuelas (Ramírez de las Casas-Deza, 1841), y su obra póstuma Anales de la ciudad de Córdoba, 1236-1850 (Ramírez de las Casas-Deza, 1948), aunque también escribió biografías, sobre la catedral de Córdoba y sobre la inquisición en esta ciudad, sobre poesía, y sobre muchos otros temas históricos y patrimoniales. Su reconocida actividad le hizo merecedor a lo largo de su vida de numerosas distinciones y dignidades, tanto en corporaciones locales como nacionales y extranjeras. Falleció el día 5 de mayo de 1874 en su ciudad natal.

\section{Interés por la Botánica}

Probablemente, su interés por la Botánica comenzó en el año 1823 cuando, tras regresar de Sevilla, quedó a vivir en casa de un familiar, entre cuyos conocidos estaba el Dr. Francisco José Camacho, que había sido Rector del Colegio de la Asunción. Este hecho propició que conociera a un sobrino de aquel, Rafael Entrenas y Camacho (1786-1835), entusiasta botánico cordobés y discípulo del agustino José de Jesús Muñoz Capilla (1771-1840). Este último era corresponsal de los botánicos Mariano Lagasca y Félix Haenseler (Laza Palacios, 1944), y creó un importante herbario -que data de 1830-, del que existe un catálogo exhaustivo (Jordano \& Ocaña, 1955 \& 1957), y que se conserva actualmente en el Herbario COFC de la Facultad de Ciencias de la Universidad de Córdoba. Su relación con Entrenas y con Muñoz Capilla debió ser muy intensa, aunque no siempre por motivos botánicos, y a la muerte del primero, Casas-Deza publicó su biografía en el Boletín Oficial de la Provincia (Ramírez de las CasasDeza, 1977). En todo caso, sus contactos con el Jardín Botánico de Madrid le hicieron a menudo mediador entre los botánicos cordobeses y José Demetrio Rodríguez ${ }^{1}$, colaborador de Mariano Lagasca, al que sucedería en la dirección de esta institución tras su muerte, en 1836.

Sobre las primeras impresiones que CasasDeza tuvo sobre la flora cordobesa hay un comentario, nada alentador, en su libro El Indicador Cordobés (1837): "En el término de Córdoba, á pesar de su feracidad, no se hallan plantas capaces de llamar la atención de los botánicos. Para formar alguna idea de lo que produce el país en esta línea, podrán ver los aficionados á este estudio los hervarios que se conservan en la oficina de Farmacia de Don Rafael Pabon², y en la conocida con el nombre de Capilla, especialmente el primero". Pero, poco a poco cambió de opinión, tal vez tras su relación con el profesor Fernando Amor y Mayor (1822-1863), compañero del Instituto Provincial de Segunda Enseñanza de Córdoba desde el año 1847, a quien menciona Ramírez de las Casas-Deza (1856) en unos párrafos en los que, probablemente por estar más documentado, expone más justamente el estado del conocimiento botánico en la provincia:

"...Posee el territorio cordobés una flora bastante estensa y variada y sobre todo hermosa, lozana y atrevida. Según las más recientes investigaciones botánicas llegan á 1500 las especies que entre fanerógamas y cryptógamas nacen en el suelo (1) Las grandes dehesas que á la abundancia de gramineas unen las leguminosas. En las orillas de los arroyos se encuentran muchos ranúnculos: en los sembrados varias abundantes viznagas, thapsias, hinojos y lobas, $y$ otras muchas umbeladas. En sitios próximos al

\footnotetext{
1 “...deseaba así mismo el Sr. Entrena, como tan aficionado a la Botánica, que yo le pusiese en relaciones con el catedrático Dr. José Demetrio Rodríguez, como en efecto lo hice. El Sr. Entrena le mandó copia del catálogo de su herbario y se remitieron mutuamente muchas semillas y cebollas de plantas. En Noviembre de 1826 me escribía diciendo: "quisiera visitase Ud. Al Sr. Rodríguez por ver si tiene listas de semillas de plantas medicinales que apetece al P. Mtro. Muñoz y las cuatro cinco que yo le pedía, e igualmente quiere designar los esqueletos de las plantas colectadas en Sierra de Segura por mi amigo y de las mías para remitírsele. También le dirá Ud. Que estamos revisando varios trozos de herbarios antiguos, compuestos de más de 800 plantas, parte colectadas por el mismo P. Mtro. El año 1822 y otras muchas determinadas por D. Rafael León..."; (Ramírez de las Casas-Deza, 1977).

${ }^{2}$ Rafael Mariano Pavón -o Pabón- fue un conocido farmacéutico cordobés. Su hijo, Francisco de Borja Pavón (18141904), farmacéutico, trabajó en la farmacia paterna, que después heredó. Fue Cronista Oficial de Córdoba, académico y escritor local, amigo personal de Ramírez de las Casas-Deza.
} 
rio, varias especíes de solanos, y en sus isletos algunas especies de salsolas: varios chenopodios, una grande y magnifica salvia de flor purpúrea, $y$ la hermosa nigela de corola azul, suelen hallarse en las veredas y lindes de los sembrados. Muchas especies de crucíferas, entre ellas la mostaza blanca y negra, y la gualda, que tiñe de amarillo, cubren estensas praderas: la gualda, que con sus flores pajizas forma agradable contraste con el intenso azul de los lirios, las borrajas y las buglosas.

De la vegetación de la sierra mucho mas variada que la de la campiña, solo nos limitaremos en este lugar á citar algunos géneros. Sus pinares están formados por el pinus pinea; los encinares por las encinas de fruto dulce y amargo, la de fruto en forma de avellana, el roble y el alcornoque. La coscoja, que produce gran cantidad de grana kermes ${ }^{3}$ y el quejigo, que dá las agalla del pais, los madroños, los brezos arboreo y de escobas, el lentisco y cornicabra, que dan hermosas raíces para la ebanistería, el durillo, cuya madera pajiza es muy parecida á la del box, las retamas, hiniestas y agracejos: estos arbustos forman el monte bajo; pero, uno de los géneros que mas parece caracterizar esta vegetación es el de las jaras, de que se encuentran muchas especies: nosotros solo citaremos algunas, como la blanca, las de hoja de salvia, de romero y de álamo, la crispa, la de mompelier y la que produce el ládano. En los montes se encuentran almezos, enebros, y aunque ya pocos, algunos algarrobos de troncos gigantescos y cerca de los arroyos, alisos, álamos y fresnos, como igualmente adelfas arboreas $y$ de hermosas flores; $y$ en otros parages acantos $y$ abundantes mirtos.

(1) [Nota a pie de página en el original] " $D e$ estas 1300 especies eran no pocas conocidas por algunos botánicos naturales de esta ciudad; pero solo ha llegado á reunir este número nuestro amigo y comprofesor el distinguido naturalista Dr. D. Fernando Amor y Mayor..."

De su interés por la botánica y los botánicos, que desea resaltarse aquí, nos quedan sus apuntes biográficos sobre la vida del destacado botánico cordobés José de Jesús Muñoz Capilla, y unos interesantes listados de plantas de algunas zonas de la provincia de Córdoba. Los primeros forman parte de un manuscrito original, encuadernado y conservado en la Biblioteca Nacional de España (Ramírez de las Casas-Deza, 1857, Ms. 5618.
BNE), donde recoge sus notas biográficas sobre treinta y seis escritores españoles de diferentes épocas, entre ellos la del polifacético botánico agustino con el que mantuvo relación. Respecto de los segundos, en la Biblioteca Provincial de Córdoba (BPC), se conservan cuatro documentos manuscritos en los que Ramírez de las CasasDeza hace una extensa relación de las Plantas del término de Córdoba, otro con una selección de ellas, un tercero en el que no explicita la procedencia, y un cuarto con plantas del término de Fuencaliente, Ciudad Real (Ramírez de las Casas-Deza, s/f. Ms. 109. BPC).

La estructura de estos documentos es muy diferente. El relativo a las plantas del término de Córdoba, el más extenso de todos, consta de 27 páginas, en las que relaciona unas 718 especies de plantas vasculares, además de un puñado de briófitos y hongos (incluidos los liquenizadores). Lo hace utilizando la nomenclatura binominal linneana, que acompaña a veces del autor que supuestamente describió la especie, y muy pocas del nombre vulgar. Aunque en los listados no hay epígrafes que delaten la clasificación seguida, claramente las plantas están ordenadas en su mayor parte de acuerdo con el sistema sexual de Linneo, al menos hasta la página 17 , en que aparece un nuevo lote de plantas ordenadas de acuerdo con el mismo sistema pero no contempladas con anterioridad. La mayor parte de ellas son plantas silvestres, pero las hay también cultivadas y ornamentales. La identificación en muchos casos es cuestionable, pues el nombre corresponde a taxones sin representación en la provincia de Córdoba (o incluso en España), explicable por el desarrollo incipiente de la botánica y las limitaciones bibliográficas. En todo caso, no cabe la menor duda de que CasasDeza no tenía un conocimiento botánico extenso que avalase su manuscrito, que debió elaborar a partir de las plantas conservadas en los herbarios de los botánicos y farmacéuticos de su época. De hecho, cita una especie expresamente del herbario de Muñoz Capilla (v.g., Moluccela laevis), y un conjunto de ellas con nombres científicos que imputa a su amigo Entrenas. El documento Plantas de Córdoba de solo tres páginas, está concebido de manera muy diferente, pues Casas-Deza relaciona tan solo 61 especies, muchas de ellas medicinales o cultivadas, por lo que cabe deducir que es una selección con tal finalidad. En este caso

\footnotetext{
${ }^{3}$ Kermes o quermes, pigmento de color carmesí que se obtiene a partir de insectos hembra del género Kermes (Hemiptera), pulgones que viven a expensas de la savia de la coscoja (Quercus coccifera) y otros árboles.
} 
anota en primer lugar la denominación vulgar, que acompaña seguidamente del nombre científico.

Los otros dos documentos poseen un especial interés pues las plantas que recoge, fundamentalmente de interés medicinal, van acompañadas de un código binario, del que el primer número se corresponde con la Clase de Linneo y el segundo, tal vez, con la caja de herbario en el que se guardaba el material. La clasificación de Linneo la empleó tanto su amigo Rafael Entrenas, como señala González Soriano (1923; [su herbario] "clasificado por el sistema de Linneo, está cuidadosamente conservado en el Instituto General y Técnico; comprende 921 especies y 24 clases y está fechado un año después de la muerte del autor: 1836"), como Muñoz Capilla, en cuyo herbario que quedó finalmente incluido aquel (Jordano \& Ocaña, 1957). Desde luego, CasasDeza no debió recolectar de manera sistemática, ni debió ser reconocido por ello, pues en el herbario de Muñoz Capilla, que se conserva en la Universidad de Córdoba, no hay planta alguna de Ramírez de las Casas-Deza, y sí de su amigo Rafael Entrenas (Jordano \& Ocaña, 1957). No obstante, tenía los conocimientos necesarios para poder incluir un esbozo botánico en sus memorias médico-geográficas, y hay constancia de que debió ayudar a la recolección de algunas plantas, como cabe deducir de una carta en la que Rafael Entrenas le describe los caracteres del género Stachys, y le insta para que intente su recolección "en las hazas y sitios pedregosos de la sierra y en las inmediaciones de esta; aunque no la he visto pasado el rio" (carta de Rafael Entrenas; Ramírez de las Casas-Deza s/f. Ms. 115. BPC). Cabría la posibilidad de que las anotaciones binarias indicadas hicieran referencia al herbario de su compañero Amor y Mayor que, al parecer, estuvo depositado en el Instituto donde trabajaban y que contenía 624 especies pertenecientes a 72 familias que, sin embargo, estaban ordenadas de acuerdo con el sistema de De Candolle ${ }^{4}$. Algunas de las plantas del herbario de Amor fueron enviadas por éste a Mariano de la Paz Graells (1809-1898), director del Museo de Historia Natural de 1851 a 1868, que comprendía el Jardín Botánico y el
Gabinete de Historia Natural, instituciones que formaban la Sección de Ciencias Naturales dentro de la Facultad de Filosofía (Montero, 2003). Se sabe que, al menos en 1851, Amor le envió "cinco colecciones, de fósiles, rocas, minerales, plantas y animales, respectivamente, hechas en Córdoba por D. Fernando Amor..." (Barreiro, 1992), pero no debió ser la única vez. De hecho, de acuerdo con la información suministrada por González Bueno (com. escrita, 2018), se tiene constancia de un paquete rotulado con letra de Graells como "Plantas de Cordoba / envio de Amor", que contenía 95 pliegos de plantas vasculares y no vasculares, ordenadas alfabéticamente por género y con etiquetas manuscritas de F. Amor ${ }^{5}$, material que se conserva actualmente en el Herbario del Real Colegio Alfonso XII de San Lorenzo de EI Escorial, Madrid (Carrasco et al., 1997).

El primero de los documentos con códigos binarios al que se ha hecho referencia consta solo de cuatro páginas, en las que Ramírez de las Casas-Deza relaciona 90 especies (más un puñado de ellas de las que solo indica el nombre vulgar), en su mayoría silvestres (¿de Córdoba?) y de interés medicinal, que refiere mediante el nombre vernáculo, seguido del científico y del código binario. Más interés, por la delimitación de la procedencia, tiene el manuscrito relativo a la Historia Natural de Fuencaliente, que debió elaborar tras su estancia en dicha población, entre mayo y octubre del año 1840. Aquí pasó un breve período de tiempo en el que se ganó el sustento como médico $\mathrm{y}$, además, con una retribución diaria sacada de lo que pagaban los "bañantes" en espera de que aceptaran su solicitud como Director de las aguas de Fuencaliente, lo que finalmente no consiguió, pero al menos durante dicho período simultaneó sus actividades con "adquirir datos para escribir una memoria sobre las aguas de esta villa y su topografía..." (Ramírez de las Casas-Deza, 1977). La primera la insertó en el Semanario Pintoresco, pero la segunda no llegó a realizarla. De esta experiencia derivan sus publicaciones sobre el lugar ( $p$. ej., Ramírez de las Casas-Deza, 1844), y probablemente también el listado de plantas al que se hace referencia.

\footnotetext{
${ }^{4}$ González Soriano (1923). Este herbario, fechado de 1845 a 1858, debió contener en algún momento una colección más importante, sobre todo si se tiene en cuenta lo que Amor y Mayor señalaba en una carta dirigida al profesor PérezArcas, el 20 de agosto de 1853: “... en lo que he trabajado mucho ha sido en plantas; las tenía un poco abandonadas, pero he dedicado a ellas todo el año. He hecho subir el número de fanerógamas a más de mil, algunas magníficas..." (Amo Serrano, 1933).

${ }^{5}$ La mayor parte no lleva fecha precisa, aparece "2a mitad de marzo", y cuando llevan año es 1852; son pocas las localidades señaladas, las herborizaciones más frecuentes proceden de Valdelashuertas, La Albaida, La Ruzafa, topónimos -todas ellas- enclavados en la proximidad de Santa María de Trassierra (Córdoba) (González Bueno, com. escrita, 2018).
} 
Este consta de tres páginas en las que relaciona 80 especies de plantas vasculares, en su mayoría silvestres y de carácter medicinal, para las que indica el nombre vulgar y el científico, y que acompaña también de un código numérico binario similar al empleado en el listado sin procedencia anteriormente mencionado.

\section{La botánica en la medicina rural y su interés para Ramírez de las Casas-Deza}

Hasta el último tercio del siglo XIX, en el que comienza a descubrirse el origen microbiano de muchas enfermedades, la explicación sobre la enfermedad de los organismos se relacionaba con multitud de causas, tanto naturales como espirituales. Entre las primeras, el medio ambiente - desde el clima a la dieta- sería considerado un factor fundamental. Desde los estudios hipocráticos hasta los de los ilustrados, persiste la idea de la importancia del medio físico y su relación con el estado de salud lo que daría lugar, a partir de finales del XVIII, a trabajos de la llamada geografía médica o topografía médica. Esta materia tenía como base la historia natural de una localidad, asociando el tipo y número de enfermedades, y el número de muertes, a la geología, botánica, zoología, clima, etc. (Urteaga, 1985; 1993). Que "algo" podía haber en el ambiente que causaba enfermedades se sabía o intuía, pero la causa no. En este sentido, el médico Agustín Espuig escribía en El Heraldo Médico de 1 de julio de 1854 un largo artículo titulado "Si el cólera es epidemia, puede tener la misma causa que ellas", en el que escribe sobre varias enfermedades y epidemias, y dice textualmente: "Los animales, las aguas, los vegetales, los minerales sueltan por las variaciones de la atmósfera álitos ó gases, que combinándose de mil maneras con el aire, inducen á los que lo respiran tanta cantidad de dolencias cuanta sea la disposición de las organizaciones;..." (Espuig, 1854). En lo concerniente a los vegetales como agentes causales, Urteaga (1993) menciona expresamente la "Historia Natural" de Buffon, donde el barón trata de explicar esta relación: " $\mathrm{La}$ tierra hace las plantas y las plantas hacen a los animales, y la tierra, las plantas y los animales hacen al hombre, pues las cualidades de los vegetales proceden inmediatamente de la tierra $y$ del aire: el temperamento y las demás cualidades de los animales hervíboros tienen mucha conexión con la de las plantas de que se nutren; $y$ finalmente, las cualidades de los animales que se alimentan de otros animales tanto como de las plantas dependen, aunque con menos inmediación, de estas mismas causas, cuya influencia se extiende hasta su índole y costumbres...".

Para llevar a cabo esta línea de trabajo naturalista, las autoridades gubernamentales crearon una Comisión Permanente de Topografías, para elaborar un documento -clave- que orientase a los médicos en cómo estructurar sus memorias, siendo las sociedades médicas las que se encargarían de distribuirlo. Las dos claves a las que se ha tenido acceso, una de 1829 (Ms. 112, BPC; Anónimo, 1829) y otra de 1831 (Ms. 115, BPC; Anónimo, 1831), muestran diferencias importantes en lo concerniente a las plantas. En la más antigua, de la "Sociedad MédicoChirurgica de Cádiz", el epígrafe que interesa se denomina "De los séres del Reino Vegetal", y al proceder se exige: "Io- Número y clasificación de los individuos de este reino segun el sistema de Linneo. $2^{\circ}$ - Enumeracion de los que son de mas frecuente cultivo y usos domesticos. $3^{\circ}$ - Designar prolijamente los que son nocivos ó venenosos". Sin embargo, en la posterior, de la Real Academia de Medicina y Cirugía de Barcelona, el epígrafe se refiere como "Reino vegetal y Agricultura", y en él se insta a anotar solo el nombre vulgar, además de lo que se cultiva, abonos, etc., las propiedades medicinales e industriales de las plantas, cómo combatir las que son nocivas, y qué enfermedades tienen, sus causas y soluciones.

Es en este contexto en el que hay que ubicar el interés de Ramírez de las Casas-Deza, de formación médico, por las plantas y animales, pero que estrictamente no puede considerarse como botánico, ni zoólogo. Sus conocimientos de estas materias son los necesarios para identificar cultivos y plantas medicinales, y tampoco de forma exhaustiva. Sin duda, Ramírez de las Casas-Deza es un claro seguidor de la teoría ambientalista, y sus libros (Corografía, Indicador, Geografía) están muy impregnados de esas ideas; la importancia que da a los baños minero-medicinales (Ramírez de las Casas-Deza, 1844) nos recuerda a lo que Casco Solís (2001) llamó "naturalismo terapéutico". López Ontiveros (1984) considera como topografías médicas todas sus publicaciones sobre las aguas de Fuencaliente (Ciudad Real), así como el manuscrito sobre la epidemia de calenturas en El Carpio (Córdoba), enviado a la Academia de Medicina de Sevilla, si bien el que más se ajusta a lo que era una topografía médica es el manuscrito sobre la topografía del partido de Pozoblanco (Ramírez de las Casas-Deza, s/f. Ms. 113. BPC), pues es aquí donde se extiende en la geografía e historia natural, señalando los vegetales nocivos, las plantas medicinales y los principales cultivos, además de los animales.

Los manuscritos reseñados, a excepción del 
más extenso (Plantas del término de Córdoba), que es una extensa relación sobre todas las plantas de las que tiene noticia en el territorio, van en esta línea, y recogen con más o menos acierto un puñado de plantas cultivadas y silvestres con interés medicinal, tal vez con la intención de efectuar una topografía médica de Córdoba, y desde luego de manera expresa, la topografía médica de Fuencaliente ("Historia Nat' de Fuencaliente"), que nunca llegó a concluir.

\section{Agradecimientos}

Nuestro agradecimiento al Prof. Antonio González Bueno por sus comentarios y aclaraciones, que han mejorado sustancialmente el original. También, agradecer a la Biblioteca Provincial de Córdoba (Junta de Andalucía) las facilidades dadas en la consulta del material manuscrito de Ramírez de las Casas-Deza allí alojado.

\section{Bibliografía}

\section{Fuentes primarias}

Ramírez de las Casas-Deza, L. M. s/f. Manuscritos $\mathrm{n}^{\circ}$ 109. Fondo antiguo. Biblioteca Provincial de Córdoba. Incluye: Plantas de Cordoba; Plantas del término de Cordoba; Plantas de ¿Cordoba?; Historia Nat. de Fuencaliente.

Ramírez de las Casas-Deza, L. M. s/f. Topografía del partido de Pozoblanco. Fondo Ramírez de las CasasDeza. Carpeta de manuscritos $n^{\circ} 113$. Biblioteca Provincial de Córdoba.

Ramírez de las Casas-Deza, L. M. (1857). Biografías de escritores españoles. Originalmente escritas ó ilustradas con nuevas noticias. Manuscrito MS 5618. Biblioteca Nacional de España.

Ramírez de las Casas-Deza, L. M. s/f. Manuscritos $\mathrm{n}^{\circ}$ 115. Fondo antiguo. Biblioteca Provincial de Córdoba. Incluye: Recortes de Prensa, Tablas y Correspondencia.

\section{Fuentes secundarias}

Amo Serrano, J. (1933). Don Fernando Amor y Mayor. Boletín de la Real Academia de Córdoba de Ciencias, Bellas Letras y Nobles Artes, 39, 265-272.

Anónimo (1829). Clave que facilita la descripción topografico-medica de un pais cualquiera, con arreglo á la cual han de trabajarla los corresponsales de la Sociedad Médico-Chirurgica de Cádiz. Fondo Ramírez de las Casas-Deza. Carpeta de manuscritos $\mathrm{n}^{\circ}$ 112. Biblioteca Provincial de Córdoba.

Anónimo (1831). Clave que la Comisión Permanente de Topografías ha propuesto a la Real Academia de Medicina y Cirugía de Barcelona para facilitar la formacion de la topografía de los pueblos que componen la provincia de Cataluña. Fondo Ramírez de las Casas-Deza. Carpeta de manuscritos $n^{\circ} 115$.
Biblioteca Provincial de Córdoba.

Espuig, A. (1854). Si el colera es epidemia, puede tener la misma causa que ellas. Correspondencia de El Heraldo Medico. 1 de julio de 1854. Fondo Ramírez de las Casas-Deza. Carpeta de manuscritos $n^{\circ} 115$. Biblioteca Provincial de Córdoba.

Barreiro, A. J. (1992). El Museo Nacional de Ciencias Naturales (1771-1935), Ed. de Pedro M. Sánchez Moreno. Museo Nacional de Ciencias Naturales Theatrum Naturae. Madrid.

Borja Pavón, F. de (1874). D. Luis María de las CasasDeza. Apuntes Necrológicos. Imprenta del Diario de Córdoba. Córdoba.

Carrasco, M. A., García, A., Perea, D. \& Martín-Blanco, J. C. (1997). Herbarium of M. P. Graells (1809-1898) discovered in the Real Colegio Alfonso XII of San Lorenzo de El Escorial, Madrid, Spain. Taxon, 46, 367-370.

Casco Solís, J. (2001). Las topografías médicas: revisión y cronología. Asclepio, LIII (1), 213-244.

González Soriano, A. (1923). Flora cordobesa. Boletín de la Real Academia de Córdoba de Ciencias, Bellas Letras y Nobles Artes, 3, 93-95; 4, 93-99; 5, 49-59; 6, 77-83.

Jordano, D. \& Ocaña, M. (1955). Catálogo del herbario de los botánicos cordobeses Rafael de León y Gálvez, Fr. José de Jesús Muñoz Capilla, Rafael Entrenas y Antonio Cabrera. Boletín de la Real Academia de Córdoba de Ciencias, Bellas Letras y Nobles Artes, 73, 35-290.

Jordano, D. \& Ocaña, M. (1957). Catálogo del herbario de los botánicos cordobeses Rafael de León y Gálvez, Fr. José de Jesús Muñoz Capilla, Rafael Entrenas y Antonio Cabrera. Anales del Instituto Botánico A. J. Cavanilles, 14, 597-715.

Laza Palacios, M. (1944). Estudios sobre la flora andaluza. Anales de la Real Academia de Farmacia (Madrid), 10, 157-199; 367-411; 497-556.

López González, G. (2005). Desde la creación del Jardín de Migas Calientes (1755) hasta A. J. Cavanilles (1801-1804). In: M. Pilar San Pío -ed.- El Real Jardín Botánico de Madrid (1755-2005). Ciencia, Colección y Escuela. Lunwerg Editores. Barcelona.

López Ontiveros, A. (1984). Topografías médicas y geografía en la obra de Casas-Deza. Boletín de la Real Academia de Ciencias, Bellas Letras y Nobles Artes de Córdoba, 106, 301-323.

Montero, A. (2003). La paleontología y sus colecciones desde el Real Gabinete de Historia Natural al Museo Nacional de Ciencias Naturales. Monografías $n^{\circ} 19$. Museo Nacional de Ciencias Naturales-CSIC. 383 págs.

Ramírez de las Casas-Deza, L. M. (1837). Indicador cordobés ó sea Manual histórico-topográfico de la ciudad de Córdoba. Imprenta de D. Rafael García Rodríguez. Córdoba.

Ramírez de las Casas-Deza, L. M. (1840). Corografía Histórico-Estadística de la provincia y obispado de Córdoba. Imprenta de Noguér y Manté. Córdoba.

Ramírez de las Casas-Deza, L. M. (1841). Breve tratado de Geografía de la Provincia de Córdoba para uso de los colegios y escuelas. Imprenta García. Córdoba.

Ramírez de las Casas-Deza, L. M. (1844). Los Baños 
de Fuen-Caliente. Semanario Pintoresco Español, 20, 157-160.

Ramírez de las Casas-Deza, L. M. (1856). Indicador cordobés, ó sea manual histórico-topográfico de la ciudad de Córdoba, ed. 3 (aumentada considerablemente). Imprenta y tipografía de D. Fausto García Tena. Córdoba.

Ramírez de las Casas-Deza, L. M. (1948). Anales de la ciudad de Córdoba, 1236-1850. Edición de la Real Academia de Córdoba. Tipografía Artística. Córdoba. [obra póstuma]

Ramírez de las Casas-Deza, L. M. (1977). Memorias de
Ramírez de las Casas Deza [Prólogo de J. M. Cuenca Toribio: Biografía y memorias especialmente literarias de don Luis María Ramírez y las Casas-Deza, entre los Arcades de Roma Ramilio Tartesiaco, individuo correspondiente de la Real Academia Española]. Universidad de Córdoba, Instituto de $\mathrm{H}^{\mathrm{a}}$ de Andalucía. 319 págs. Córdoba.

Urteaga, L. (1985). Higienismo y ambientalismo en la medicina decimonónica. Acta Hispanica ad Medicinae Scientiarumque Historiam Ilustrandam, 5-6, 417-425.

Urteaga, L. (1993). La teoría de los climas y los orígenes del ambientalismo. GeoCrítica, 99, 1-36. 